\title{
Article \\ Organic Emitters Showing Excited-States Energy Inversion: An Assessment of MC-PDFT and Correlation Energy Functionals Beyond TD-DFT
}

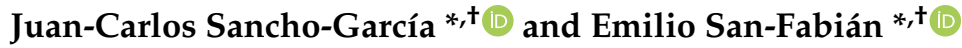 \\ Department of Physical Chemistry, University of Alicante, E-03080 Alicante, Spain \\ * Correspondence: jc.sancho@ua.es (J.-C.S.-G.); sanfa@ua.es (E.S.-F.) \\ † These authors contributed equally to this work.
}

Citation: Sancho-García, J.C.; San-Fabián,E. Violation of Hund's Rule in Organic Emitters: An Assessment of MC-PDFT and Correlation Energy Functionals Beyond TD-DFT. Computation 2022, 10, 13. https://doi.org/10.3390/ computation 10020013

Academic Editor: Henry Chermette

Received: 23 December 2021

Accepted: 12 January 2022

Published: 18 January 2022

Publisher's Note: MDPI stays neutral with regard to jurisdictional claims in published maps and institutional affiliations.

Copyright: (c) 2022 by the authors. Licensee MDPI, Basel, Switzerland. This article is an open access article distributed under the terms and conditions of the Creative Commons Attribution (CC BY) license (https:// creativecommons.org/licenses/by/ $4.0 /)$.

\begin{abstract}
The lowest-energy singlet $\left(S_{1}\right)$ and triplet $\left(T_{1}\right)$ excited states of organic conjugated chromophores are known to be accurately calculated by modern wavefunction and Time-Dependent Density Functional Theory (TD-DFT) methods, with the accuracy of the latter heavily relying on the exchange-correlation functional employed. However, there are challenging cases for which this cannot be the case, due to the fact that those excited states are not exclusively formed by single excitations and/or are affected by marked correlation effects, and thus TD-DFT might fall short. We will tackle here a set of molecules belonging to the azaphenalene family, for which research recently documented an inversion of the relative energy of $S_{1}$ and $T_{1}$ excited states giving rise to a negative energy difference $\left(\Delta E_{S T}\right)$ between them and, thereby, contrary to most of the systems thus far treated by TD-DFT. Since methods going beyond standard TD-DFT are not extensively applied to excited-state calculations and considering how challenging this case is for the molecules investigated, we will prospectively employ here a set of non-standard methods (Multi-Configurational Pair Density Functional Theory or MC-PDFT) and correlation functionals (i.e., Lie-Clementi and Colle-Salvetti) relying not only on the electronic density but also on some modifications considering the intricate electronic structure of these systems.
\end{abstract}

Keywords: TD-DFT; MC-PDFT; Lie-Clementi; Colle-Salvetti; OLEDs

\section{Introduction}

The violation of Hund's rule in molecules [1], analogously to atoms, is commonly ascribed to an inversion of the excitation energies of the lowest states of spin-singlet $\left(S_{1}\right)$ or spin-triplet $\left(T_{1}\right)$ multiplicity. In common situations, the energy difference between $S_{1}$ and $T_{1}$ excited states, that is $\Delta E_{S T}=E\left(S_{1}\right)-E\left(T_{1}\right)$ is positive, contrary to what happens if Hund's rule is altered (in that case, $\Delta E_{S T}$ would be negative). Note that the negative sign contradicts the fact that the exchange energy $(K)$ is normally thought to be positive, historically $\Delta E_{S T} \approx 2 K$, thus, implying that the lowest singlet excited state lies energetically above the lowest triplet excited state and not the opposite.

That exchange energy is known to be of the order of hundreds of meV for common organic chromophores, and this becomes a key parameter for photophysics and related applications [2-4]. However, it has been demonstrated that strong correlation effects can decrease the $\Delta E_{S T}$ value [5,6] and even invert the energies of $S_{1}$ and $T_{1}$ excited states due to a more marked stabilization of the former vs. the latter state [7], although very few molecules are discovered up to now displaying such an excited-state energy inversion. Additionally, fast environmental effects (whenever they are reliably introduced) could also lead to negative $\Delta E_{S T}$ values.

Among those environmental effects, we mention thermal fluctuations of molecular conformations or microscopic electronic polarization effects in amorphous films of carbazole derivatives [8], thus, opening a whole world of future studies and applications around this 
unexpected issue. However, for solvation effects, current implementations of continuum solvation models should be employed with caution since it could lead to spurious excitedstate energy inversion [9].

The physical origin of this inversion and its possible practical implications should not merely be considered as an academic questions. As an example of practical use, due to the spin statistics, triplet excitons (dark) are known to be formed upon a 3:1 ratio with respect to singlet excitons (bright) thus limiting the efficiency of electroluminiscent processes. Therefore, the mentioned energy inversion could be further exploited in the, e.g., recovery of triplet excitons created upon electroluminiscence to increase the internal quantum efficiency or quantum yields of Organic Light-Emitting Diodes (OLEDs).

Many other applications in related fields (photocatalysts, covalent organic frameworks, liquid crystals, etc.) have also been envisioned and recently reviewed [10]. However, from an experimental point of view, the range of disclosed molecules showing that excitedstate energy inversion is very limited, and this goes back to the discovery in the 1980s of some azaphenalene molecules candidates [11,12] for such a violation of Hund's rule. Those initial molecules were also extended to other (not-yet-synthesized) candidates after a massive screening of compounds recently performed [13], showing that the topic is still open and under active investigation.

Therefore, theoretical methods based on one-electron excitations (i.e., Time-Dependent Density Functional Theory or TD-DFT) are questioned in its current implementation to recognize that excited-state energy inversion, due to the lack of inclusion of higher-thansingle excitations into their formulation. In contrast, wavefunction methods have been shown in recent studies [14-21] to provide reasonably accurate values for that $\Delta E_{S T}$ energy difference, although at a cost certainly higher than TD-DFT.

That limitation of TD-DFT is known to occur independently of the underlying exchangecorrelation functional and basis sets chosen. However, since excited-state wavefunction methods can capture double and higher-order excitations by definition, depending of the truncation done for the excitation operator, those methods are able to predict the excitedstate energy inversion while concomitantly providing accurate individual (i.e., $S_{1}$ and $T_{1}$ ) energies for the excited-states involved.

Based on these findings, our goal here is to investigate if methods going beyond standard (TD-)DFT could predict that excited-state energy inversion and thus compete in accuracy with wavefunction results. To assess that, we will employ methods merging wavefunction and correlation functionals, in the hope of including both kind of correlation effects (dynamical and non-dynamical) for any of the electronic states involved. These results will also serve to confirm the key role played by marked correlation effects, as well as to invigorate more research of DFT methods out of the most commonly found implementations.

\section{Systems, Methods, and Computational Details}

\subsection{Choice of the Target Systems}

The set of systems selected is exclusively motivated by the previous experimental and theoretical findings mentioned above. The set of azaphenalene molecules shown in Figure 1 is known to display negative $\Delta E_{S T}$ values at various wavefunction levels, from pioneering studies [14-16] later extended to related and/or larger systems [17-21], which clearly constitutes a challenge for any theoretical method.

Interestingly, chemical substitution of the heptazine core $-\mathrm{C}_{6} \mathrm{H}_{7} \mathrm{H}_{3}-$ with chlorine $-\mathrm{C}_{6} \mathrm{H}_{7} \mathrm{Cl}_{3}-$, cyano $-\mathrm{C}_{6} \mathrm{H}_{7}(\mathrm{CN})_{3}-$, or p-methoxyphenylene $-\mathrm{C}_{6} \mathrm{~N}_{7}\left(\mathrm{p}-\mathrm{C}_{6} \mathrm{H}_{4} \mathrm{OCH}_{3}\right)_{3}-$ groups preserved the negative $\Delta E_{S T}$ value, showing that the chemical structure of the core is indeed responsible as well as that more potential and synthetically viable molecules could soon be theoretically disclosed and/or experimentally achieved. 
<smiles>C1=CC2=CC=CC3=CC=CC(=C1)N23</smiles>

MAP<smiles>C1=CC2=NC=CC3=NC=CC(=N1)N23</smiles>

$4 \mathrm{AP}$<smiles>C1=CC2=NC=NC3=NC=NC(=C1)N23</smiles>

$5 \mathrm{AP}$<smiles>C1=NC2=NC=NC3=NC=NC(=N1)N23</smiles>

$7 A P$

Figure 1. Chemical structures (from left to right) of the molecules MAP (monoazaphenalene or cyclazine), TAP (tetraazaphenalene), 5AP (pentaazaphenalene), and 7AP (heptaazaphenalene or heptazine). Hydrogen atoms are omitted for clarity.

From the experimental point of view, some studies that were performed in the 1980s also concluded with a real possibility of having an excited-state inversion for some of these azaphenalenes [11,12]. More recently, some heptazine derivatives have been successfully employed as emitters in real devices [22,23], with an exceptionally high quantum yield reported and explained by the (partial) conversion of triplet into singlet excitons possibly assisted by a negative $\Delta E_{S T}$ value.

Note also that other related cores could be also potential candidates, after the conclusions reached by a massive screening of thousands of potential azaphenalene candidates by Pollice et al. [13]. However, we are more interested in assessing the reliability of theories going beyond (TD-)DFT and we will, thus, restrict this work to the compounds shown in Figure 1 for which reference results are available as well.

\subsection{Physical Meaning of Reduced Density Matrices}

While the electronic density, or first-order reduced density matrix, is given by:

$$
\rho(\mathbf{r})=N \int \Psi^{\star}\left(\mathbf{r}, \mathbf{r}_{2}, \ldots, \mathbf{r}_{N}\right) \Psi\left(\mathbf{r}, \mathbf{r}_{2}, \ldots, \mathbf{r}_{N}\right) d \mathbf{r}_{2} \ldots d \mathbf{r}_{N},
$$

and thus integrates over the number of electrons $N, \int \rho(\mathbf{r}) d \mathbf{r}=N$, the corresponding spinless second-order reduced density matrix integrates to the total number of interacting electron pairs:

$$
\iint \rho_{2}\left(\mathbf{r}_{1}, \mathbf{r}_{2}\right) d \mathbf{r}_{1} d \mathbf{r}_{2}=\frac{N(N-1)}{2},
$$

and represents the probability density of finding a particle at point $\mathbf{r}_{1}$ and simultaneously another particle at point $\mathbf{r}_{2}$. The explicit form is given by:

$$
\gamma_{2}\left(\mathbf{x}_{1}^{\prime}, \mathbf{x}_{2}^{\prime} ; \mathbf{x}_{1}, \mathbf{x}_{2}\right)=\frac{N(N-1)}{2} \int \ldots \int \gamma_{N}\left(\mathbf{x}_{1}^{\prime}, \mathbf{x}_{2}^{\prime}, \mathbf{x}_{3} \ldots, \mathbf{x}_{N} ; \mathbf{x}_{1}, \mathbf{x}_{2}, \mathbf{x}_{3}, \ldots, \mathbf{x}_{N}\right) d \mathbf{x}_{3} \ldots d \mathbf{x}_{N},
$$

or better its reduced form $\xi_{2}\left(\mathbf{r}_{1}^{\prime}, \mathbf{r}_{2}^{\prime} ; \mathbf{r}_{1}, \mathbf{r}_{2}\right)=\sum_{s_{1}, s_{2}}=\left.\gamma_{2}\left(\mathbf{x}_{1}^{\prime}, \mathbf{x}_{2}^{\prime} ; \mathbf{x}_{1}, \mathbf{x}_{2}\right)\right|_{s_{i}^{\prime}=s_{i}}$. Finally, the diagonal element or $\rho_{2}\left(\mathbf{r}_{1}, \mathbf{r}_{2}\right)$ would account from any correlation effect arising from interparticle interaction, as it can be easily seen from the electron-electron mean value as a function of this new magnitude:

$$
\left\langle\hat{V}_{e e}\right\rangle=\iint \frac{\rho_{2}\left(\mathbf{r}_{1}, \mathbf{r}_{2}\right)}{\left|\mathbf{r}_{1}-\mathbf{r}_{2}\right|} d \mathbf{r}_{1} d \mathbf{r}_{2}
$$

Note also that $\rho(\mathbf{r})$ and $\rho_{2}\left(\mathbf{r}_{1}, \mathbf{r}_{2}\right)$ are related through:

$$
\rho(\mathbf{r})=\frac{2}{N-1} \int \rho_{2}\left(\mathbf{r}_{1}, \mathbf{r}_{2}\right) d \mathbf{r}_{2} .
$$

In the following, we will denote $\rho_{2}(\mathbf{r})=\left.\rho_{2}\left(\mathbf{r}_{1}, \mathbf{r}_{2}\right)\right|_{\mathbf{r}_{1}=\mathbf{r}_{2}}$ as the function at the twoelectron coalescence point, whose modelling has been extensively pursued in the past [24-26], as well as its integration into excited-state formalisms $[27,28]$, as the next step for the description of electronic structure beyond the use of merely the electronic density $\rho(\mathbf{r})$. 


\subsection{Theories Going beyond (TD-)DFT}

The methods included in this study can be categorized in popular language as methods going beyond (standard) DFT, in the sense that they are based on the on-top second-order reduced density matrix $\rho_{2}(\mathbf{r})$, and not only on the first-order density $\rho(\mathbf{r})$, or post-MCSCF, in the sense that a Multi-Configurational Self-Consistent Field (MCSCF) calculations needs to be done first from which the magnitude $\rho_{2}(\mathbf{r})$ is obtained.

Note that the on-top second-order reduced density matrix represents the probability that two opposite-spin electrons are found at point $\mathbf{r}$ and integrates to the total number of interacting pairs. Multiconfiguration Pair-Density Functional Theory (MC-PDFT [29-32]) can be thus viewed as a post-MCSCF method that evaluates the energy of any state with on-top pair-density function theory. Basically, for a MCSCF wavefunction, $\left|\Psi^{\mathrm{MCSCF}}\right\rangle=$ $\sum_{\mu} C_{\mu}\left|\Psi_{\mu}\right\rangle$, one can obtain the total electronic energy as:

$$
E=\left\langle\Psi^{\mathrm{MCSCF}}|\hat{T}| \Psi^{\mathrm{MCSCF}}\right\rangle+\int v(\mathbf{r}) \rho(\mathbf{r}) d \mathbf{r}+\iint \frac{\rho\left(\mathbf{r}_{1}\right) \rho\left(\mathbf{r}_{2}\right)}{\left|\mathbf{r}_{1}-\mathbf{r}_{2}\right|} d \mathbf{r}_{1} d \mathbf{r}_{2}+E_{x c}\left[\rho(\mathbf{r}), \rho_{2}(\mathbf{r})\right],
$$

with the different terms being the kinetic, potential, Coulomb, and exchange-correlation energies, respectively, the latter relying on a modification of a common DFT functional to be employed together with Equation (1). The most extensively tested on-top density functional is called tPBE and will, thus, be used here consequently.

On the other hand, one can also directly employ a functional explicitly depending upon the design of the on-top second-order reduced density matrix, such as the Colle-Salvetti (CS [33-35]) correlation functional. We will denote, in the following, the CS expression as a two-body correlation functional, cast as $E_{c}\left[\rho(\mathbf{r}), \rho_{2}(\mathbf{r})\right]$, in contrast with conventional or one-body functionals commonly used for standard DFT calculations, or simply $E_{c}[\rho(\mathbf{r})]$. Note that the famous Lee-Yang-Parr (LYP [36]) correlation functional is a reformulation of the Colle-Salvetti expression to avoid the explicit dependence on $\rho_{2}(\mathbf{r})$ at the price of neglecting its use with, e.g., MCSCF wavefunctions. For this case, the total electronic energy is calculated by a two-step procedure,

$$
E=\left\langle\Psi^{\mathrm{MCSCF}}\left|\hat{T}+\hat{V}_{\mathrm{Ne}}+\hat{V}_{e e}\right| \Psi^{\mathrm{MCSCF}}\right\rangle+E_{c}\left[\rho(\mathbf{r}), \rho_{2}(\mathbf{r})\right],
$$

after adding the correlation (mainly dynamic) energy to the energy calculated by the underlying MCSCF procedure, which already includes the non-dynamic (or static) correlation energy, with $\hat{T}, \hat{V}_{\mathrm{Ne}}, \hat{V}_{e e}$ the kinetic, nuclear-electron, and electron-electron operators.

Another not-so-common approximation is given by the Lie-Clementi (LC [37,38]) correlation functional, with an explicit dependence on the natural (fractional) occupation numbers, if a MCSCF calculation is done first. A modified density is built, such as

$$
\rho_{m}(\mathbf{r})=\sum_{i} n_{i} e^{-\left(2-n_{i}\right)^{2} / 2} \tilde{\rho}(\mathbf{r}),
$$

depending on the density built from the natural orbitals, $\tilde{\rho}(\mathbf{r})$, and the corresponding natural orbital occupation numbers $\left(n_{i}\right)$. That density is, thus, inserted into the reparameterized correlation functional of Gombas et al., generally denoting this class of functionals as $E_{c}\left[\rho_{m}(\mathbf{r})\right]$. Interestingly, those orbitals with $n_{i}<2$ do not contribute to the correlation energy as much as those doubly occupied $\left(n_{i}=2\right)$, thus, describing both ground- and excited-states independently.

Therefore, MC-PDFT, $E_{x c}\left[\rho(\mathbf{r}), \rho_{2}(\mathbf{r})\right]$, and $E_{c}\left[\rho_{m}(\mathbf{r})\right]$ exchange-correlation functionals will be all based here on a Complete Active Space Self-Consistent Field (CASSCF) wavefunction with an active space of $N$ electrons housed in $M$ orbitals, or simply $(N, M)$, to incorporate non-dynamic (or static) correlation effects in a consistent way. The active space chosen, $(6,6)$ or $(12,12)$, is indeed based on the occupancy (and degeneracy) or molecular orbitals found at the uncorrelated level. 
Note that: (i) MC-PDFT could be instead used with many MCSCF wavefunction types, such as GVB, CASSCF, RASSCF, CAS-CI, and RAS-CI, as is the case for the other two-body functionals too. We will, however, limit this work to the same wavefunction type for both schemes for the sake of coherence. (ii) Since these theories can be applied to any state of interest, independently of its spin, there is no need to invoke a linear-response regime as it happens for TD-DFT. (iii) Any of these methods will incorporate all correlation effects, be they static or dynamic, thus, possibly disentangling the importance of any of these contributions into the final results.

\subsection{Computational Details}

The ground-state $\left(S_{0}\right)$ geometry of all the compounds was optimized by the B97-3c method [39], without any imaginary frequency obtained. The energy difference between the lowest-energy spin-singlet $\left(S_{1}\right)$ and spin-triplet $\left(T_{1}\right)$ excited-states is denoted as $\Delta E_{S T}$, which is normally positive unless for an energy inversion of the $S_{1}$ and $T_{1}$ energies, thus, giving rise to $\Delta E_{S T}<0$. The def2-SVP and def2-TZVP basis sets [40] are used for all the calculations, with the auxiliary def2/JK and def2-TZVP/C basis sets [41] to reduce the computational cost.

For some control TD-DFT calculations, we will employ the $\omega$ B97, $\omega$ B97X [42], and $\omega \mathrm{B} 97 \mathrm{X}-2$ [43], which form a set of range-separated exchange-correlation functionals belonging, respectively, to non-hybrid ( $\omega$ B97), hybrid ( $\omega$ B97X), and double-hybrid ( $\omega$ B97X-2) rungs to infer if the addition of exact-exchange (for a hybrid) or pertubation-like (for a doublehybrid) brings any difference to the results.

We used the following quantum-chemical packages for the calculations performed here: ORCA 5.0 [44] for the (standard) TD-DFT with hybrid and double-hybrid functionals, GAMESS [45] for the MC-PDFT method with the tPBE functional, and an in-house program $[46,47]$ (interfaced with GAMESS) for the $E_{c}\left[\rho_{m}(\mathbf{r})\right]$ and $E_{c}\left[\rho(\mathbf{r}), \rho_{2}(\mathbf{r})\right]$ calculations employing the Lie-Clementi and the Colle-Salvetti correlation functionals.

\section{Results and Discussion}

\subsection{Reference Results Available}

We compare, in the following, the results obtained here not only with previously applied wavefunction methods but also with respect to the experimental information available: Leupin et al. obtained [11] for MAP a $S_{1} \leftarrow S_{0}$ and $T_{1} \leftarrow S_{0}$ excitation energies of 0.972 and between 0.972 and 0.984 , respectively, and thus with a $\Delta E_{S T}$ energy difference possibly negative. For 4AP, Leupin et al. obtained [12] excitation energies below 2.39 for $S_{1} \leftarrow S_{0}$ (although the final value could not be completely determined experimentally) and $2.29 \mathrm{eV}$ for $T_{1} \leftarrow S_{0}$, respectively, again not excluding a $\Delta E_{S T}<0$ value depending on how low $S_{1} \leftarrow S_{0}$ was in reality.

Finally, for 7AP, Leonard et al. obtained [48] a $S_{1} \leftarrow S_{0}$ excitation energy of $2.60 \mathrm{eV}$. From the theoretical point of view, a large number of previous studies are available. However, some of them (DLPNO-STEOM-CCSD, NEVPT2, SCS-CC2, and SCS-ADC(2)) used the def2-TZVP basis set, as done here, but others (EOM-CCSD, DLPNO-NEVPT2, ADC(2), and ADC(3)) employed the smaller cc-pVDZ or def2-SVP basis sets instead.

In all cases, all these correlated wavefunction methods were able to predict a $\Delta E_{S T}<0$ value for all the MAP, 4AP, 5AP, and 7AP systems. Choosing one of these methods as reference, necessarily motivated by the completeness of the values found in the literature [17] together with the high accuracy demanded, and the SCS-CC2/def2-TZVP results are listed next for cross-comparison along the study.

MAP: $\quad S_{1} \leftarrow S_{0}\left(T_{1} \leftarrow S_{0}\right)$ value of $1.110(1.334) \mathrm{eV}$, with $\Delta E_{S T}=-0.22 \mathrm{eV}$

4AP: $\quad S_{1} \leftarrow S_{0}\left(T_{1} \leftarrow S_{0}\right)$ value of $2.258(2.342) \mathrm{eV}$, with $\Delta E_{S T}=-0.08 \mathrm{eV}$

5AP: $\quad S_{1} \leftarrow S_{0}\left(T_{1} \leftarrow S_{0}\right)$ value of $2.308(2.541) \mathrm{eV}$, with $\Delta E_{S T}=-0.23 \mathrm{eV}$

7AP: $\quad S_{1} \leftarrow S_{0}\left(T_{1} \leftarrow S_{0}\right)$ value of $2.847(3.226) \mathrm{eV}$, with $\Delta E_{S T}=-0.38 \mathrm{eV}$ 


\subsection{TD-DFT Calculations}

First, we also illustrate here if a standard TD-DFT calculation is able or not to provide a $\Delta E_{S T}<0$ value and (if so) with what accuracy. For this purpose, we will choose not a selection of different functionals but a set of models of increasing complexity (i.e., semilocal, hybrid, and double-hybrid functionals). Additionally, since range-separation was before invoked as an accurate tool for the modelling of organic emitters of this type [49], Table 1 gathers the results obtained by the $\omega \mathrm{B} 97, \omega \mathrm{B} 97 \mathrm{X}$, and $\omega \mathrm{B} 97 \mathrm{X}-2$ range-separated exchange-correlation functionals.

Strikingly, $\omega \mathrm{B} 97$ and $\omega \mathrm{B} 97 \mathrm{X}$ are unable to predict the excited-state energy inversion, providing values for individual excitation energies not differing greatly between both methods, which is not often the case since those values are known to depend on the amount of (short-range) exact-exchange introduced ( $0 \%$ in $\omega$ B97 vs. $\approx 16 \%$ in $\omega \mathrm{B} 97 \mathrm{X})$ and /or the range-separation parameter ( $\omega=0.4$ in $\omega$ B97 vs. $\omega=0.3$ in $\omega$ B97X).

Furthermore, we note how the $S_{1} \leftarrow S_{0}$ excitation energies are overestimated (severely in the case of 7AP) by both methods with respect to experimental or SCS-CC2 reference results. Note that many previous publications [14-21] already demonstrated how this was the case for all exchange-correlation functionals assessed up to now, which is also confirmed here.

We additionally assessed if the use of the $\omega$ B97X-D3 or $\omega$ B97X-D4 models, which are reparameterized to include the dispersion correction, would lead to any difference: the $\Delta E_{S T}$ value only changed only $0.01 \mathrm{eV}$ with respect to the value calculated by the original $\omega$ B97X.

We next analysed the performance of the $\omega \mathrm{B} 97 \mathrm{X}-2$ double-hybrid functional, which, in its extension to excited states [50,51], includes a contribution from double excitations, thus, going beyond the single excitations introduced by TD-DFT routinely. We can observe how this method actually predicts $\Delta E_{S T}<0$ values between -0.4 and $-0.7 \mathrm{eV}$, roughly speaking that are, thus, too large with respect to the reference results. An additional concern arises from the inspection of individual $S_{1} \leftarrow S_{0}$ and $T_{1} \leftarrow S_{0}$ excitation energies, since the method seems to severely underestimate (by up to $1 \mathrm{eV}$ ) the former values progressively as a function of the $\mathrm{N}$ atoms introduced into the chemical structure.

The overestimation of the $T_{1} \leftarrow S_{0}$ excitation energies is slightly attenuated with respect to the $S_{1} \leftarrow S_{0}$ ones but again with values deviating too much with respect to the reference results. Shortly speaking, although this method is able to provide negative values for $\Delta E_{S T}$, the results appear to be affected by a systematic error. The use of double-hybrid functionals has been recently and more systematically examined [52], with some of the models assessed being promising enough to display accurate individual excitation energies: we thus refer the reader to that study for further information and confirmation about the key role played by double excitations into the final values.

\subsection{MC-PDFT Calculations}

We will inspect the CASSCF results shown in Table 2 to first observe the effect of using both basis sets, def2-SVP and def2-TZVP, for this set of calculations. For the CASSCF(6,6) results, going from def2-SVP to def2-TZVP implies a slight increase of the $S_{1} \leftarrow S_{0}$ and $T_{1} \leftarrow S_{0}$ excitation energies, with the exception of the latter for the 7AP molecule, but asymmetrically, with the corresponding $\Delta E_{S T}$ values altered significantly. For the $\operatorname{CASSCF}(12,12)$ case, the variations for 7AP are also significant and deviate from the SCS-CC2/def2-TZVP reference values.

For MAP, the CASSCF $(6,6) /$ def2-TZVP calculation already provided close results to reference SCS-CC2/def2-TZVP results, with $S_{1} \leftarrow S_{0}$ and $T_{1} \leftarrow S_{0}$ excitation energies differing by 0.15 and $0.10 \mathrm{eV}$, respectively, and thus leading to a negative $\Delta E_{S T}$ value of -0.18 compared to $-0.22 \mathrm{eV}$ as reference. However, a larger active space is not definitively giving any advantage here, as it was also found before [17], stabilizing too much the $S_{1}$ $\left(T_{1}\right)$ state and leading consequently to an overly negative (positive) $\Delta E_{S T}$ value with the def2-SVP (def2-TZVP) basis set. 
Table 1. Vertical excitation energies and associated $\Delta E_{S T}$ energy difference (all in $\mathrm{eV}$ ) calculated with different methods.

\begin{tabular}{|c|c|c|c|c|}
\hline Molecule & Method & $S_{1} \leftarrow S_{0}$ & $T_{1} \leftarrow S_{0}$ & $\Delta E_{S T}$ \\
\hline \multirow{3}{*}{ MAP } & $\omega \mathrm{B} 97$ & 1.420 & 1.195 & 0.26 \\
\hline & $\omega \mathrm{B} 97 \mathrm{X}$ & 1.385 & 1.167 & 0.22 \\
\hline & $\omega \mathrm{B} 97 \mathrm{X}-2$ & 0.791 & 1.166 & -0.38 \\
\hline \multirow{3}{*}{$4 \mathrm{AP}$} & $\omega \mathrm{B} 97$ & 2.695 & 2.199 & 0.41 \\
\hline & $\omega \mathrm{B} 97 \mathrm{X}$ & 2.531 & 2.150 & 0.38 \\
\hline & $\omega \mathrm{B} 97 \mathrm{X}-2$ & 1.642 & 2.092 & -0.45 \\
\hline \multirow{3}{*}{$5 \mathrm{AP}$} & $\omega \mathrm{B} 97$ & 2.752 & 2.381 & 0.37 \\
\hline & $\omega \mathrm{B} 97 \mathrm{X}$ & 2.687 & 2.335 & 0.35 \\
\hline & $\omega \mathrm{B} 97 \mathrm{X}-2$ & 1.650 & 2.298 & -0.65 \\
\hline \multirow{3}{*}{ 7AP } & $\omega \mathrm{B} 97$ & 3.391 & 3.132 & 0.26 \\
\hline & $\omega \mathrm{B} 97 \mathrm{X}$ & 3.310 & 3.059 & 0.26 \\
\hline & $\omega \mathrm{B} 97 X-2$ & 1.933 & 2.592 & -0.66 \\
\hline
\end{tabular}

For $4 \mathrm{AP}$, the agreement is not so close to $\mathrm{CASSCF}(6,6)$, with both excitation energies largely overestimated (by $0.3-0.7 \mathrm{eV}$ ) independently of the basis set chosen. A larger active space, CASSCF(12,12), seems beneficial only with the def2-SVP basis set, which appears to indicate a not so balanced treatment of correlation effects in the absence of a dynamical correlation correction.

This overestimation was also found for 5AP and 7AP, particularly striking for the latter and again independently of the active space fixed. Overall, it seems that the CASSCF results do not suffice to lead to accurate and robust results by themselves, although negative $\Delta E_{S T}$ values are mostly obtained. Previous publications show how the need of dynamic correlation effects (i.e., NEVPT2) as a further step to obtain more accurate and robust results $[17,18]$.

Table 2. Excited-state energies and associated $\Delta E_{S T}$ energy difference (all in eV) calculated with the CASSCF method.

\begin{tabular}{cclccr}
\hline Basis Set & Molecule & Method & $\boldsymbol{S}_{\mathbf{1}} \leftarrow \boldsymbol{S}_{\mathbf{0}}$ & $\boldsymbol{T}_{\mathbf{1}} \leftarrow \boldsymbol{S}_{\mathbf{0}}$ & $\boldsymbol{\Delta} \boldsymbol{E}_{\boldsymbol{S T}}$ \\
\hline \multirow{6}{*}{ def2-SVP } & MAP & CASSCF(6,6) & 1.218 & 1.436 & -0.22 \\
& 4AP & CASSCF(6,6) & 2.554 & 2.803 & -0.25 \\
& 5AP & CASSCF(6,6) & 2.686 & 2.916 & -0.23 \\
& 7AP & CASSCF(6,6) & 3.896 & 4.217 & -0.32 \\
\cline { 2 - 6 } & MAP & CASSCF(12,12) & 0.145 & 0.696 & -0.55 \\
& 4AP & CASSCF(12,12) & 2.214 & 2.358 & -0.14 \\
& 5AP & CASSCF(12,12) & 2.581 & 2.519 & 0.06 \\
& 7AP & CASSCF(12,12) & 2.752 & 3.210 & -0.46 \\
\hline \multirow{5}{*}{ def2-TZVP } & MAP & CASSCF(6,6) & 1.256 & 1.427 & -0.17 \\
& 4AP & CASSCF(6,6) & 2.964 & 2.864 & 0.10 \\
& 5AP & CASSCF(6,6) & 2.995 & 3.068 & -0.07 \\
& 7AP & CASSCF(6,6) & 5.237 & 4.437 & 0.80 \\
\cline { 2 - 6 } & MAP & CASSCF(12,12) & 0.179 & 0.722 & -0.54 \\
& 4AP & CASSCF(12,12) & 1.977 & 2.171 & -0.19 \\
& 5AP & CASSCF(12,12) & 2.762 & 2.688 & 0.07 \\
& 7AP & CASSCF(12,12) & 4.334 & 4.637 & -0.30 \\
\hline
\end{tabular}

The use of the tPBE correlation functional together with Equation (7) is presented next in Table 3, again for both basis sets (def2-SVP and def2-TZVP) and both active spaces of the underlying CASSCF calculation. For MAP, the CASSCF $(6,6)+t P B E$ results are considerably accurate with both basis sets, not only for the target $\Delta E_{S T}$ energy difference but also for the 
individual excitation energies. The use of the larger $\operatorname{CASSCF}(12,12)$ active space instead increases both excitation energies, especially the former, and reverse the sign of $\Delta E_{S T}$.

For 4AP and 5AP, the CASSCF $(12,12)+\mathrm{tPBE}$ results are very accurate with the def2TZVP basis set and with respect to the SCS-CC2 reference values. For 7AP, the CASSCF $(12,12)$ $+\mathrm{tPBE}$ results are also relatively accurate, leading to a negative $\Delta E_{S T}$ value with both basis sets and correcting the overestimation of values found at the CASSCF $(12,12)$ level with the def2-TZVP basis set. It thus appears that the addition of a (modified) correlation functional is qualitatively beneficial; however, more research is still needed to confirm the application of MC-PDFT to other chromophores and related systems.

Table 3. Excited-state energies and associated $\Delta E_{S T}$ energy difference (all in eV) calculated with MC-PDFT.

\begin{tabular}{|c|c|c|c|c|c|}
\hline Basis Set & Molecule & Method & $S_{1} \leftarrow S_{0}$ & $T_{1} \leftarrow S_{0}$ & $\Delta E_{S T}$ \\
\hline \multirow{8}{*}{ def2-SVP } & MAP & $\operatorname{CASSCF}(6,6)+\mathrm{tPBE}$ & 1.168 & 1.284 & -0.12 \\
\hline & $4 \mathrm{AP}$ & $\operatorname{CASSCF}(6,6)+\mathrm{tPBE}$ & 2.135 & 1.871 & 0.26 \\
\hline & $5 \mathrm{AP}$ & $\operatorname{CASSCF}(6,6)+\mathrm{tPBE}$ & 2.153 & 2.437 & -0.28 \\
\hline & 7AP & $\operatorname{CASSCF}(6,6)+\mathrm{tPBE}$ & 2.715 & 3.155 & -0.44 \\
\hline & MAP & $\operatorname{CASSCF}(12,12)+\mathrm{tPBE}$ & 1.523 & 1.463 & 0.06 \\
\hline & $4 \mathrm{AP}$ & $\operatorname{CASSCF}(12,12)+\mathrm{tPBE}$ & 2.181 & 2.713 & -0.53 \\
\hline & $5 \mathrm{AP}$ & $\operatorname{CASSCF}(12,12)+\mathrm{tPBE}$ & 2.720 & 2.889 & -0.17 \\
\hline & 7AP & $\operatorname{CASSCF}(12,12)+\mathrm{tPBE}$ & 2.849 & 3.373 & -0.52 \\
\hline \multirow{8}{*}{ def2-TZVP } & MAP & $\operatorname{CASSCF}(6,6)+\mathrm{tPBE}$ & 1.191 & 1.209 & -0.02 \\
\hline & $4 \mathrm{AP}$ & $\operatorname{CASSCF}(6,6)+\mathrm{tPBE}$ & 2.038 & 1.865 & 0.17 \\
\hline & $5 \mathrm{AP}$ & $\operatorname{CASSCF}(6,6)+\mathrm{tPBE}$ & 1.140 & 2.769 & -1.63 \\
\hline & 7AP & $\operatorname{CASSCF}(6,6)+\mathrm{tPBE}$ & 4.641 & 3.325 & 1.32 \\
\hline & MAP & $\operatorname{CASSCF}(12,12)+\mathrm{tPBE}$ & 1.515 & 1.438 & 0.08 \\
\hline & $4 \mathrm{AP}$ & $\operatorname{CASSCF}(12,12)+\mathrm{tPBE}$ & 2.420 & 2.572 & -0.15 \\
\hline & $5 \mathrm{AP}$ & $\operatorname{CASSCF}(12,12)+\mathrm{tPBE}$ & 2.304 & 2.579 & -0.17 \\
\hline & 7AP & $\operatorname{CASSCF}(12,12)+\mathrm{tPBE}$ & 1.906 & 2.148 & -0.24 \\
\hline
\end{tabular}

\subsection{Lie-Clementi (LC) and Colle-Salvetti (CS) Calculations}

These two functionals are here applied with the $\operatorname{CASSCF}(6,6)$ active space and the def2-SVP basis set to avoid a known (and long-standing) problem with these methods, i.e., the double counting of the dynamical correlation energy, which might be minimized using the smallest admissible active space in the underlying CASSCF calculations [53,54].

Table 4 presents these pioneering results, from which interesting features can be observed: (i) a negative $\Delta E_{S T}$ is provided in all cases, contrarily to some of the former methods and the isolated CASSCF $(6,6)$ calculations of Table 2, which clearly underlines the major role played by dynamical correlation effects for describing at least quantitatively these states; (ii) the cost-effective Lie-Clementi functional overestimates the individual excitation energies, systematically increasing them with respect to the CASSCF $(6,6)$ values; and (iii) the Colle-Salvetti functional, relying on the introduction of the $\rho_{2}(\mathbf{r})$ variable, provides closer values to reference results, again with the exception of 7AP for which these are still overestimated.

Given the variety of methods tested, Figure 2 reports the calculated $\Delta E_{S T}$ energy difference for a better comparison. Contrary to previous TD-DFT applications, and with very few exceptions, the figure clearly shows how it is possible to obtain negative values (i.e., inverted $S_{1}$ and $T_{1}$ excitation energies) with these methods thanks to the combination of non-dynamical and dynamical correlation effects. 
Table 4. Excited-state energies and associated $\Delta E_{S T}$ energy difference (all in eV) calculated with non-standard correlation functionals.

\begin{tabular}{|c|c|c|c|c|c|}
\hline Basis Set & Molecule & Method & $S_{1} \leftarrow S_{0}$ & $T_{1} \leftarrow S_{0}$ & $\Delta E_{S T}$ \\
\hline \multirow{8}{*}{ def2-SVP } & \multirow{2}{*}{ MAP } & $\operatorname{CASSCF}(6,6)+\mathrm{LC}$ & 1.737 & 1.941 & -0.20 \\
\hline & & $\operatorname{CASSCF}(6,6)+\mathrm{CS}$ & 0.968 & 1.364 & -0.40 \\
\hline & \multirow{2}{*}{$4 \mathrm{AP}$} & $\operatorname{CASSCF}(6,6)+\mathrm{LC}$ & 3.116 & 3.386 & -0.27 \\
\hline & & $\operatorname{CASSCF}(6,6)+\mathrm{CS}$ & 2.501 & 2.931 & -0.43 \\
\hline & \multirow{2}{*}{$5 \mathrm{AP}$} & $\operatorname{CASSCF}(6,6)+\mathrm{LC}$ & 3.273 & 3.453 & -0.18 \\
\hline & & $\operatorname{CASSCF}(6,6)+C S$ & 2.543 & 3.043 & -0.50 \\
\hline & \multirow{2}{*}{ 7AP } & $\operatorname{CASSCF}(6,6)+\mathrm{LC}$ & 4.568 & 4.892 & -0.32 \\
\hline & & $\operatorname{CASSCF}(6,6)+\operatorname{CS}$ & 3.554 & 4.017 & -0.46 \\
\hline
\end{tabular}

Taking into account the use here of the def2-SVP basis set, we can next compare these results with the high-quality values available in literature: (i) For MAP, NEVPT2(6,6)/def2SVP results [18] gave $S_{1} \leftarrow S_{0}$ and $T_{1} \leftarrow S_{0}$ excitation energies of 1.102 and $1.319 \mathrm{eV}$ with the CASSCF $(6,6)+C S$ results differing by only 0.13 and $0.05 \mathrm{eV}$, respectively. (ii) For 5AP, EOM-CCSD/cc-pVDZ results [13] are 2.251 and $2.329 \mathrm{eV}$ for $S_{1} \leftarrow S_{0}$ and $T_{1} \leftarrow S_{0}$ excitation energies, respectively, with the CASSCF $(6,6)+\mathrm{tPBE}$ results of 2.153 and $2.437 \mathrm{eV}$ differing by only 0.10 and $0.11 \mathrm{eV}$, respectively. (iii) For 7AP, DLPNO-NEVPT2(6,6)/def2SVP results [13] led to $S_{1} \leftarrow S_{0}$ and $T_{1} \leftarrow S_{0}$ excitation energies of 2.552 and $2.906 \mathrm{eV}$ with the CASSCF $(12,12)+\mathrm{tPBE}$ results being the closest ones but still differing by 0.30 and $0.47 \mathrm{eV}$, respectively.

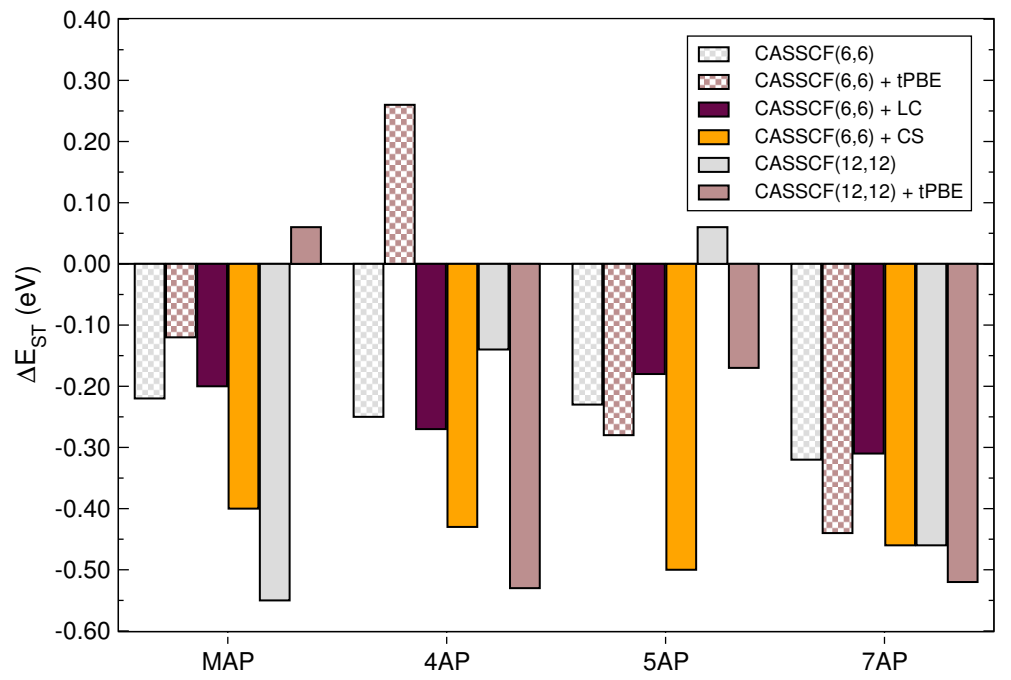

Figure 2. $\Delta E_{S T}$ values for the molecules selected (with the def2-SVP basis set).

\section{Conclusions}

The field of (TD-)DFT has so impressively advanced over the recent decades thanks to the two-fold and concurring efforts of continuously merging developments and applications. As a corollary, the latter would have not been possible without major advances from developments, often considered part of basic but completely needed Science. In this regard, new methods have historically been fostered by providing cost-effective yet accurate expressions and implementations for wide applications or, on the other hand, by tackling cutting-edge applications at the frontier of knowledge to move the field forward. In other words, inaccurate results are often needed to question why (TD-)DFT behaves as it does and how it can be rigorously and systematically improved.

Therefore, we attempted to continue building that interface between both worlds (developments and applications) by selecting a long-standing chemical problem of revisited interest: the energy inversion of the lowest spin-singlet and spin-triplet excited states of 
azaphenalene compounds intended to be used as organic emitters or photocatalysts. For that purpose, knowing from previous works than TD-DFT was not a reliable path to adequately address this issue, we applied methods employing not only the electronic density as the sole ingredient of an exchange-correlation functional but also other more involved magnitudes, e.g., the on-top pair density.

Overall, the use of the latter into the MC-PDFT scheme or as part of the explicit formulation of the Colle-Salvetti correlation functional offers an attractive way to overcome the limitations found for (TD-)DFT, although at a higher computational cost. However, we are also aware that further research is needed to benchmark these non-conventional methods as well as to reduce their computational cost and scaling with the system size, for which more challenging applications will be also welcome in the near future.

\section{Concluding Remarks: A Personal Note}

We would like to contribute with this article, as part of the Special Issue in honour of Professor Karlheinz Schwarz on the occasion of his 80th birthday, to celebrate the outstanding role played by Professor Schwarz in the field of Density Functional Theory (DFT) through his scientific career [55]. The authors met him as part of the International Scientific Committee of the "International Conference on Density-Functional Theory and its Applications", which is likely one of the longest-lived events to exist in the fields of theoretical and computational Chemistry and Physics after Paris (1995), Vienna (1997), Rome (1999), Madrid (2001), Brussels (2003), Geneva (2005), Amsterdam (2007), Lyon (2009), Athens (2011), Durham (2013), Debrecen (2015), Tällberg (2017), and Alicante (2019) editions.

Thanks to the strong activity of Heinz promoting DFT worldwide, we had the opportunity to enjoy, as local organizers of the last edition (see Figure 3), his compromise and illusion with this series of conferences. This work is, thus, our small recognition to his talented, vibrant, active, and kind figure, promoting DFT worldwide alongside an outstanding scientific career.

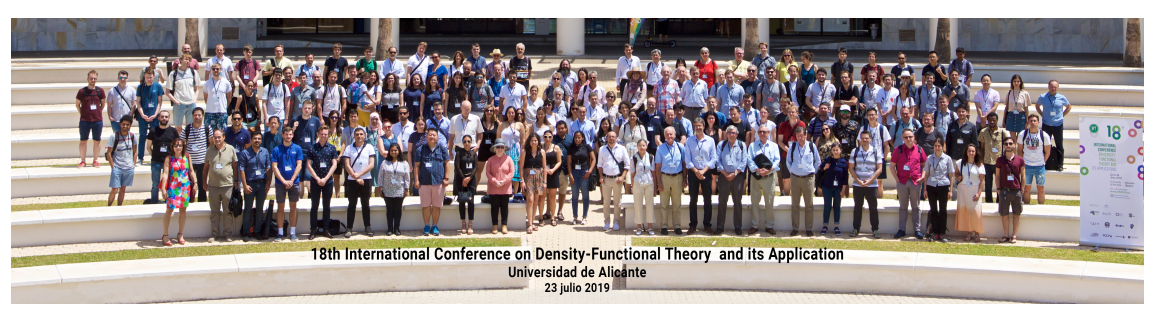

Figure 3. Group picture taken along the "18th International Conference on Density-Functional Theory and its Applications", celebrated (2019) in the campus of the University of Alicante. Professor Karlheinz Schwarz is standing on the first row (11th starting from the right to the left side).

Author Contributions: Conceptualization, J.-C.S.-G. and E.S.-F.; methodology, J.-C.S.-G. and E.S.-F.; software, J.-C.S.-G. and E.S.-F.; validation, J.-C.S.-G. and E.S.-F.; formal analysis, J.-C.S.-G. and E.S.-F.; investigation, J.-C.S.-G. and E.S.-F.; resources, J.-C.S.-G. and E.S.-F.; data curation, J.-C.S.-G. and E.S.-F.; writing, J.-C.S.-G. and E.S.-F.; funding acquisition, J.-C.S.-G. and E.S.-F. All authors have read and agreed to the published version of the manuscript.

Funding: This research was funded by "Ministerio de Ciencia e Innovación" of Spain grant number PID2019-106114GB-I00.

Acknowledgments: We kindly thank Chermette for the invitation to contribute to this Special Issue. We very much appreciate working together along the years with close colleagues, such as A.J. PérezJiménez (U. Alicante, Spain), Y. Olivier (U. Namur, Belgium), E. Brémond (U. Paris, France), and C. Adamo (ChimieParis Tech, France), as well as with outstanding young researchers, such as G. Ricci (U. Namur, Belgium), for developments and applications of DFT to challenging problems, such as those treated here.

Conflicts of Interest: The authors declare no conflict of interest. 


\section{References}

1. Liu, S.; Langenaeker, W. Hund's multiplicity rule: A unified interpretation. Theor. Chem. Acc. 2003, 110, 338-344. [CrossRef]

2. Köhler, A.; Beljonne, D. The singlet-triplet exchange energy in conjugated polymers. Adv. Funct. Mater. 2004, 14, 11-18. [CrossRef]

3. Gierschner, J.; Cornil, J.; Egelhaaf, H.J. Optical bandgaps of $\pi$-conjugated organic materials at the polymer limit: Experiment and theory. Adv. Mater. 2007, 19, 173-191. [CrossRef]

4. Köhler, A.; Bässler, H. Triplet states in organic semiconductors. Mater. Sci. Eng. R Rep. 2009, 66, 71-109. [CrossRef]

5. Becke, A.D. Singlet-triplet splittings from the virial theorem and single-particle excitation energies. J. Chem. Phys. 2018, 148, 044112. [CrossRef] [PubMed]

6. Becke, A.D. Communication: Optical gap in polyacetylene from a simple quantum chemistry exciton model. J. Chem. Phys. 2018, 149, 081102. [CrossRef]

7. Kollmar, H.; Staemmler, V. Violation of Hund's rule by spin polarization in molecules. Theor. Chim. Acta 1978, 48, 223-239. [CrossRef]

8. Olivier, Y.; Yurash, B.; Muccioli, L.; D’Avino, G.; Mikhnenko, O.; Sancho-García, J.C.; Adachi, C.; Nguyen, T.Q.; Beljonne, D. Nature of the singlet and triplet excitations mediating thermally activated delayed fluorescence. Phys. Rev. Mater. 2017, 1, 075602. [CrossRef]

9. Dhali, R.; Phan Huu, D.A.; Terenziani, F.; Sissa, C.; Painelli, A. Thermally activated delayed fluorescence: A critical assessment of environmental effects on the singlet-triplet energy gap. J. Chem. Phys. 2021, 154, 134112. [CrossRef]

10. Audebert, P.; Kroke, E.; Posern, C.; Lee, S.H. State of the Art in the Preparation and Properties of Molecular Monomeric s-Heptazines: Syntheses, Characteristics, and Functional Applications. Chem. Rev. 2021, 121, 2515-2544. [CrossRef]

11. Leupin, W.; Wirz, J. Low-lying electronically excited states of cycl[3.3.3]azine, a bridged $12 \pi$-perimeter. J. Am. Chem. Soc. 1980, 102, 6068-6075. [CrossRef]

12. Leupin, W.; Magde, D.; Persy, G.; Wirz, J. 1,4,7-Triazacycl[3.3.3]azine: Basicity, photoelectron spectrum, photophysical properties. J. Am. Chem. Soc. 1986, 108, 17-22. [CrossRef]

13. Pollice, R.; Friederich, P.; Lavigne, C.; dos Passos Gomes, G.; Aspuru-Guzik, A. Organic molecules with inverted gaps between first excited singlet and triplet states and appreciable fluorescence rates. Matter 2021, 4, 1654-1682. [CrossRef]

14. de Silva, P. Inverted singlet-triplet gaps and their relevance to thermally activated delayed fluorescence. J. Phys. Chem. Lett. 2019, 10, 5674-5679. [CrossRef]

15. Ehrmaier, J.; Rabe, E.J.; Pristash, S.R.; Corp, K.L.; Schlenker, C.W.; Sobolewski, A.L.; Domcke, W. Singlet-triplet inversion in heptazine and in polymeric carbon nitrides. J. Phys. Chem. A 2019, 123, 8099-8108. [CrossRef]

16. Sandoval-Salinas, M.E.; Carreras, A.; Casanova, D. Triangular graphene nanofragments: Open-shell character and doping. Phys. Chem. Chem. Phys. 2019, 21, 9069-9076. [CrossRef]

17. Ricci, G.; San-Fabián, E.; Olivier, Y.; Sancho-García, J.C. Singlet-triplet excited-state inversion in heptazine and related molecules: Assessment of TD-DFT and ab initio methods. ChemPhysChem 2021, 22, 553-560. [CrossRef] [PubMed]

18. Sanz-Rodrigo, J.; Ricci, G.; Olivier, Y.; Sancho-Garcia, J.C. Negative Singlet-Triplet Excitation Energy Gap in Triangle-Shaped Molecular Emitters for Efficient Triplet Harvesting. J. Phys. Chem. A 2021, 125, 513-522. [CrossRef] [PubMed]

19. Pios, S.; Huang, X.; Sobolewski, A.L.; Domcke, W. Triangular boron carbon nitrides: An unexplored family of chromophores with unique properties for photocatalysis and optoelectronics. Phys. Chem. Chem. Phys. 2021, 23, 12968-12975. [CrossRef] [PubMed]

20. Bhattacharyya, K. Can TDDFT render the electronic excited states ordering of Azine derivative? A closer investigation with DLPNO-STEOM-CCSD. Chem. Phys. Lett. 2021, 779, 138827. [CrossRef]

21. Sobolewski, A.L.; Domcke, W. Are Heptazine-Based Organic Light-Emitting Diode Chromophores Thermally Activated Delayed Fluorescence or Inverted Singlet-Triplet Systems? J. Phys. Chem. Lett. 2021, 12, 6852-6860. [CrossRef]

22. Li, J.; Nakagawa, T.; MacDonald, J.; Zhang, Q.; Nomura, H.; Miyazaki, H.; Adachi, C. Highly efficient organic light-emitting diode based on a hidden thermally activated delayed fluorescence channel in a heptazine derivative. Adv. Mater. 2013, 25, 3319-3323 [CrossRef]

23. Li, J.; Zhang, Q.; Nomura, H.; Miyazaki, H.; Adachi, C. Thermally activated delayed fluorescence from ${ }^{3} \mathrm{n} \pi^{*}$ to ${ }^{1} \mathrm{n} \pi^{*}$ up-conversion and its application to organic light-emitting diodes. Appl. Phys. Lett. 2014, 105, 98. [CrossRef]

24. Moscardó, F.; San-Fabián, E. Density-functional formalism and the two-body problem. Phys. Rev. A 1991, 44, 1549. [CrossRef]

25. Moscardó, F.; San-Fabián, E. A density functional for the correlation energy, deduced in the framework of the correlation factor approach. Int. J. Quantum Chem. 1991, 40, 23-32. [CrossRef]

26. Moscardó, F.; Pérez-Jiménez, Á.J. Self-consistent field calculations using two-body density functionals for correlation energy component: I. Atomic systems. J. Comput. Chem. 1998, 19, 1887-1898. [CrossRef]

27. Pastorczak, E.; Pernal, K. Electronic excited states from the adiabatic-connection formalism with complete active space wave functions. J. Phys. Chem. Lett. 2018, 9, 5534-5538. [CrossRef]

28. Pastorczak, E.; Hapka, M.; Veis, L.; Pernal, K. Capturing the dynamic correlation for arbitrary spin-symmetry CASSCF reference with adiabatic connection approaches: Insights into the electronic structure of the tetramethyleneethane diradical. J. Phys. Chem. Lett. 2019, 10, 4668-4674. [CrossRef]

29. Hoyer, C.E.; Ghosh, S.; Truhlar, D.G.; Gagliardi, L. Multiconfiguration pair-density functional theory is as accurate as CASPT2 for electronic excitation. J. Phys. Chem. Lett. 2016, 7, 586-591. [CrossRef] [PubMed] 
30. Gagliardi, L.; Truhlar, D.G.; Li Manni, G.; Carlson, R.K.; Hoyer, C.E.; Bao, J.L. Multiconfiguration pair-density functional theory: A new way to treat strongly correlated systems. Acc. Chem. Res. 2017, 50, 66-73. [CrossRef]

31. Stoneburner, S.J.; Truhlar, D.G.; Gagliardi, L. Transition metal spin-state energetics by MC-PDFT with high local exchange. J. Phys. Chem. A 2020, 124, 1187-1195. [CrossRef] [PubMed]

32. Lykhin, A.O.; Truhlar, D.G.; Gagliardi, L. Dipole Moment Calculations Using Multiconfiguration Pair-Density Functional Theory and Hybrid Multiconfiguration Pair-Density Functional Theory. J. Chem. Theory Comput. 2021, 17, 7586-7601. [CrossRef]

33. Colle, R.; Salvetti, O. Approximate calculation of the correlation energy for the closed shells. Theor. Chim. Acta 1975, 37, 329-334. [CrossRef]

34. Colle, R.; Salvetti, O. Approximate calculation of the correlation energy for the closed and open shells. Theor. Chim. Acta 1979, 53, 55-63. [CrossRef]

35. Colle, R.; Salvetti, O. Generalization of the Colle-Salvetti correlation energy method to a many-determinant wave function J. Chem. Phys. 1990, 93, 534-544. [CrossRef]

36. Lee, C.; Yang, W.; Parr, R.G. Development of the Colle-Salvetti correlation-energy formula into a functional of the electron density. Phys. Rev. B 1988, 37, 785. [CrossRef]

37. Lie, G.C.; Clementi, E. Study of the electronic structure of molecules. XXI. Correlation energy corrections as a functional of the Hartree-Fock density and its application to the hydrides of the second row atoms. J. Chem. Phys. 1974, 60, 1275-1287. [CrossRef]

38. Lie, G.C.; Clementi, E. Study of the electronic structure of molecules. XXII. Correlation energy corrections as a functional of the Hartree-Fock type density and its application to the homonuclear diatomic molecules of the second row atoms. J. Chem. Phys. 1974, 60, 1288-1296. [CrossRef]

39. Brandenburg, J.G.; Bannwarth, C.; Hansen, A.; Grimme, S. B97-3c: A revised low-cost variant of the B97-D density functional method. J. Chem. Phys. 2018, 148, 064104. [CrossRef]

40. Weigend, F.; Ahlrichs, R. Balanced basis sets of split valence, triple zeta valence and quadruple zeta valence quality for $\mathrm{H}$ to $\mathrm{Rn}$ : Design and assessment of accuracy. Phys. Chem. Chem. Phys. 2005, 7, 3297-3305. [CrossRef]

41. Weigend, F. Hartree-Fock exchange fitting basis sets for H to Rn. J. Comput. Chem. 2008, 29, 167-175. [CrossRef] [PubMed]

42. Chai, J.D.; Head-Gordon, M. Long-range corrected hybrid density functionals with damped atom-atom dispersion corrections. Phys. Chem. Chem. Phys. 2008, 10, 6615-6620. [CrossRef] [PubMed]

43. Chai, J.D.; Head-Gordon, M. Long-range corrected double-hybrid density functionals. J. Chem. Phys. 2009, 131, 174105. [CrossRef]

44. Neese, F. Software update: The ORCA program system, version 4.0. Wiley Interdiscip. Rev. Comput. Mol. Sci. 2018, 8, e1327. [CrossRef]

45. Barca, G.M.J.; Bertoni, C.; Carrington, L.; Datta, D.; De Silva, N.; Deustua, J.E.; Fedorov, D.G.; Gour, J.R.; Gunina, A.O.; Guidez, E.; et al. Recent developments in the general atomic and molecular electronic structure system. J. Chem. Phys. 2020, 152, 154102. [CrossRef]

46. Pérez-Jordá, J.M.; San-Fabián, E.; Moscardó, F. Spectroscopic constants of diatomic molecules computed correcting Hartree-Fock or general-valence-bond potential-energy curves with correlation-energy functionals. Phys. Rev. A 1992, 45, 4407. [CrossRef] [PubMed]

47. Moscardó, F.; Muñoz-Fraile, F.; Pérez-Jiménez, A.J.; Pérez-Jordá, J.M.; San-Fabián, E. Improvement of multiconfigurational wave functions and energies by correlation energy functionals. J. Phys. Chem. A 1998, 102, 10900-10902. [CrossRef]

48. Halpern, A.M.; Rossman, M.A.; Hosmane, R.S.; Leonard, N.J. Photophysics of the $\mathrm{S}_{1}$ tautm. $\mathrm{S}_{O}$ transition in tri-s-triazine. J. Phys. Chem. 1984, 88, 4324-4326. [CrossRef]

49. Penfold, T.J. On predicting the excited-state properties of thermally activated delayed fluorescence emitters. J. Phys. Chem. C 2015, 119, 13535-13544. [CrossRef]

50. Grimme, S.; Neese, F. Double-hybrid density functional theory for excited electronic states of molecules. J. Chem. Phys. 2007, 127, 154116. [CrossRef]

51. Ottochian, A.; Morgillo, C.; Ciofini, I.; Frisch, M.J.; Scalmani, G.; Adamo, C. Double hybrids and time-dependent density functional theory: An implementation and benchmark on charge transfer excited states. J. Comput. Chem. 2020, 41, 1242-1251. [CrossRef] [PubMed]

52. Sancho-Garcia, J.C.; Bremond, E.; Ricci, G.; Pérez-Jiménez, Á.J.; Olivier, Y.; Adamo, C. Violation of Hund's Rule in Molecules: Predicting the Excited-State Energy Inversion by TD-DFT with Double-Hybrid Methods. J. Chem. Phys. 2021, 156. [CrossRef]

53. Sancho-García, J.; Moscardó, F. Usefulness of the Colle-Salvetti model for the treatment of the nondynamic correlation. J. Chem. Phys. 2003, 118, 1054-1058. [CrossRef]

54. Moscardó, F.; San-Fabián, E.; Pastor-Abia, L. The Colle-Salvetti wavefunction revisited: A comparison between three approaches for obtaining the correlation energy. Theor. Chem. Acc. 2006, 115, 334-342. [CrossRef]

55. Blaha, P.; Schwarz, K.; Tran, F.; Laskowski, R.; Madsen, G.K.; Marks, L.D. WIEN2k: An APW+ lo program for calculating the properties of solids. J. Chem. Phys. 2020, 152, 074101. [CrossRef] 